\title{
The Common Fault Diagnosis Method of Diesel Engine
}

\author{
Zhibin Wang*, Yu Zhao, Jian Yan and Jiafeng Xu \\ Communication NCO Academy of Army Engineering University, Chongqing, China \\ ${ }^{*}$ Corresponding author
}

\begin{abstract}
With the rapid development of diesel engine technology, the request of the fault diagnostic technology is getting much higher. This paper describes the common fault diagnosis method of diesel engine which is used to ensure operational safety, performance, reasonable maintenance, cost reduction, pollution prevention and information collection. Moreover the development trend of diesel engine fault diagnosis technology is predicted after analyzing the common fault diagnosis method.
\end{abstract}

Keywords-diesel engine; fault diagnosis; performance parameter; oil analysis; vibration noise; instantaneous speed

\section{INTRODUCTION}

As one of the most commonly used power equipment, the diesel engine plays a decisive role in the national economy and daily life, which has been widely used in various industries such as industry, agriculture, military, construction, transportation and transportation and has become an indispensable key equipment in some industries. It has many interrelated components, complex movement, poor working conditions and other characteristics resulting in large possibility of fault which will affect the normal operation of mechanical systems, directly or indirectly, causing enormous economic losses.

The diesel engine has a complicated structure and many parts work under harsh conditions of high temperature, high pressure, high load and strong vibration, which makes the diesel engine have a higher fault rate and a large maintenance cost. Statistics show that the expenditure on maintenance can achieve to $15 \% \sim 30 \%$ among the various usage fees for diesel engine[1]. Moreover, in the event of fault, it will also affect the normal operation of the entire mechanical system, directly or indirectly, causing huge economic losses and even endanger personal safety. Therefore, the development of diesel engine fault diagnosis technology is very necessary to ensure operational safety, performance, reasonable maintenance, cost reduction, pollution prevention and information collection.

\section{COMMON FAUlt Diagnosis MethoD}

There are a variety of methods for condition monitoring and fault diagnosis of diesel engine, some of which will be introduced briefly below.

\section{A. Performance Parameter Diagnosis Method}

This method uses the performance parameters of the diesel engine to get condition monitoring and fault diagnosis in the internal diesel engine operation, which is mainly determined by the degree of combustion process that is affected by the thermal, fluid and mechanical aspects of each subsystem.

A case is studied by Aleksandar Vencl to analysis diesel engine crankshaft journal bearings failures[2]. Wear as a tribological process has major influence on the reliability and life of engine crankshaft bearings. The importance of field examinations of bearing failures due to wear is very well known. They point to the possible causes of wear and to the necessary treatment for its reduction or elimination. They obtain the results by examining 616 crankshaft bearings, damaged by different mechanisms. The bearings were installed in high-speed diesel engines, and were gathered for two years, during general repairs of the engines (overhaul), i.e. after 3000-5000 working hours. After the examination of the bearings, the fault tree analysis (FTA) was performed to determine the root causes for engine bearing failures. Each type of damage that was identified was illustrated with an appropriate high-resolution photograph. The investigations show that the basic and most conspicuous types of damage which cause bearing failures are abrasive, adhesive and surface fatigue wear. They also consider the effects of the place of installation and type of bearing material in respect to each type of wear.

Justin Flett presents the development of a fault detection and diagnosis (FDD) system for use with a diesel internal combustion engine (ICE) valve train[3]. A novel feature is generated for each of the valve closing and combustion impacts. Deformed valve spring faults and abnormal valve clearance faults were seeded on a diesel engine instrumented with one accelerometer. Five classification methods were implemented experimentally and compared. The FDD system using the Naïve-Bayes classification method produced the best overall performance, with a lowest detection accuracy and a lowest classification accuracy for the spring faults occurring on individual valves.

In order to solve the problem that the diesel engine PT fuel system is unable to field maintain, Xin-jun Wang develops a portable signal acquisition and analysis system for diesel engine PT fuel system[4]. The PT pump work Principle was obtained by analyzing the PT pump failure mapping relation between reason and failure phenomenon was analyzed. The 
diesel engine PT pump failure fuel pressure characteristics were analyzed. Using the portable signal acquisition and analysis system to diagnose the diesel engine PT fuel system, experiment results show that the system can correctly detect the diesel engine PT fuel system state.

\section{B. Oil Analysis Method}

During the operation of the diesel engine, the fault caused by component wear and tear caused is very common. Oil test analysis method is to analyze the physical and chemical properties and wear particles of the lubricating oil samples of the diesel engine to evaluate the wear of the internal combustion engine, so as to forecast its operation trend.

Antonio Sala presents the basic characteristics of a prototype fuzzy expert system being developed in order to improve the performance of a binary-logic based software on the particular application of oil analysis of Diesel engines (for industrial and transport fleet use)[5]. The system allows for reasoning under absent or imprecise measurements, providing with an interval-valued diagnostic of the suspected severity of a particular fault. A set of so-called metarules complements the basic fault dictionary for fine tuning, allowing extra functionality such as linear transformations of membership prior to logic operations, discarding previous conclusions in some circumstances and introducing the possibility of detecting contradiction between different diagnostic alternatives for the same fault. The full system is, at this moment, in development and prototype testing stage, but its preliminary results are be promising.

Wear has important, negative effects on the functioning of engine parts[6]. Additionally, this situation is very difficult to evaluate accurately in oil analysis for engine condition monitoring. Original Equipment Manufacturers (OEM), lubricant suppliers and oil analysis laboratories provide specific guidelines for wear metal concentrations. These limits provide good general guidelines for interpreting oil analysis data, but do not take into account common factors that influence the concentration of wear debris and contaminants in an oil sample. These factors involve oil consumption, fresh oil additions, etc., and particular features such as engine age, type of service, environmental conditions, etc. An improved maintenance program for internal combustion engines based on oil analysis is developed to enable a more accurate wear determination from engine oil samples.

B Leal tries to determine, by means of techniques based on Artificial Intelligence, the actual possibilities of using spectrometric analysis, done periodically to the engine oil, as predictors of the condition of the engine[7]. Some similar works have led to a simple linear regression model, but in the particular case of this work it is intended to evaluate if the specific environmental conditions of the region and the low use of aircrafts allow discussing the convenience of making use of the same criterion or elaborating some specific variant.

\section{Vibration Noise Method}

Vibration noise method is to monitor the vibration and noise of the diesel engine, extracting the characteristic parameters of the fault, in order to diagnose whether the diesel engine breaks down and where the fault position is.

Chiatti Giancarlo put forward an experimental methodology to obtain information about the in-cylinder pressure development during the combustion process of a small displacement diesel engine by using the acoustic emission[8]. He arises from two considerations. On one end, an efficient control of the combustion process has demonstrated to be crucial to ensure complying with increasingly emerging diesel emission standards and to reduce fuel consumption. On the other end, even if nowadays the employment of in-cylinder pressure sensors in combustion control techniques appears to be a very promising technology, solutions based on nonintrusive methodologies offer the indubitable advantages of guaranteeing the absence of any interaction with the engine operation. Furthermore, these solutions allow the sensor to be installed in any kind of production engine without the need of modification. As a result, the engine noise emissions have been analyzed with the aim to extract the combustion related contribution. Particular attention has been devoted to the definition of indicators able to characterize both the noise and the in-cylinder pressure development. The relationship between these indices in the entire engine operative field allowed to evaluate the combustion properties by using only the sound radiation signal as input.

Ensuring the availability and reliability of the Emergency Diesel Generators (EDGs) in nuclear power plants is a critical aspect to guarantee the plant safe shutdown in case of an emergency scenario[9]. An effective engine diagnosis method is essential to fulfill that goal. For that purpose, Jorge Arroyo presents a fast and automatic engine diagnosis method based on a single parameter: the vibration/AE signals energy. The method is based on the comparison of the vibration and $\mathrm{AE}$ energy with reference values to determine whether the engine condition is faulty. The method was applied in a test engine, and proved to work satisfactorily. Therefore, it was used to diagnose the EDGs in a nuclear power plant, where regular rigorous inspections are effected periodically. An injector fault was detected thanks to the diagnosis method.

Yong Cheng from Shandong University introduces the applications of vibration signal on internal combustion engines inspection[10]. In order to obtain accurate vibration signals, the sampling frequency, measuring points arrangement mode and sensor assembling modes are discussed. The vibration signal can be applied to diagnose some failures of internal combustion engines, including valve train, fuel injection system and piston, etc. We come to a conclusion that the technical data of combustion process can be obtained by analyzing vibration signal. The vibration signal is used to offer the reference for inspecting the combustion state of internal combustion engines, and it provides reference signal for the electronic management of internal combustion engines.

\section{Instantaneous Speed Method}

As the measurement technology advances and the accuracy of measurement equipment increases, the crankshaft speed measurement result is more and more accurate. The fluctuation of crankshaft speed embodies the change of torsion caused by 
the firing of each cylinder of the diesel engine. The study of the shaft speed signal can obtain useful information for diagnosing the working state of diesel engine.

M. Desbazeille analyzes the crankshaft angular speed variationsto monitor large diesel engines[11]. He focuses on a powerful 20-cylinder diesel engine with crankshaft natural frequencies within the operating speed range. The angular speed variations are modeled at the crankshaft free end including modeling both the crankshaft dynamical behavior and the excitation torques. As the engine is very large, the first crankshaft torsional modes are in the low frequency range.and an automated diagnosis based on an artificially intelligent system is proposed. An experimental fuel leakage fault is successfully diagnosed, including detection and localization of the faulty cylinder, as well as the approximation of the fault severity.

D. Taraza thinks that the speed variation of the crankshaft may be easily and accurately measured using a shaft encoder and counting the pulses of the internal clock of the data acquisition system[12]. If the crankshaft would be a rigid body, the variation of its angular speed could be directly correlated to the total gas-pressure torque. Actually, the variation of the crank shaft's speed has a complex nature being influenced by the torsional stiffness of the cranks, the mass moments of inertia of the reciprocating masses and the average speed and load of the engine. Analyzing only the lower harmonic orders of the speed variation spectrum can filter out the distortions produced by the dynamic response of the crankshaft. The information carried by these harmonic orders permits to establish correlations between measurements and the average gas pressure torque of the engine, and to detect malfunctions and identify faulty cylinders.

Internal-combustion engine instantaneous speed is nonstationary signal, so the empirical mode decomposition(EMD) method is used by Junlin Zheng in timefrequency analysis of instantaneous speed signal, and the signal is decomposed into several intrinsic mode functions and residual trend terms[13]. Hilbert transform is made for each of intrinsic mode function to obtain instantaneous frequency and amplitude (Hilbert spectrum), and boundary-spectrum is obtain from Hilbert spectrum. The instantaneous speed are measured of 6-135 type diesel in normal status and fault status, and the EMD analysis outcome shows that the boundary-spectrum may indicate fault's exist, and the instantaneous frequency and residual trend terms may indicate which cylinder have fault. So the EMD method can achieve the fault diagnosis very well, and offer a new method for internal-combustion engine fault diagnosis based on instantaneous speed.

\section{CONCLUSION}

This article describes the common fault diagnosis method of diesel engine. The diesel engine fault diagnosis technology should be toward non-disintegration, high-precision and intelligent direction $\backslash$. The future of diesel engine fault diagnosis is no longer a single subject, but interdisciplinary and multidisciplinary issues. With the structure of the diesel engine becoming more and more complex and the number of components and parts increasing, the proportion of mathematical statistics, multi-information fusion and artificial intelligence in the fault diagnosis system will be greater.

\section{REFERENCES}

[1] C. Wei, W. Zhang, Y. Ge. Advanced internal combustion engine. Beijing: Beijing Institute of Technology Press, 2001:154

[2] A. Vencl, A. Rac. Diesel engine crankshaft journal bearings failures: Case study. Engineering Failure Analysis, 2014, 44:217-228

[3] J. Flett, G. Bone. Fault detection and diagnosis of diesel engine valve trains. Mechanical Systems and Signal Processing, 2016, 72: 316-327

[4] X. Wang, Y. Cai, X. Lin. Diesel Engine PT Pump Fault Diagnosis Based on the Characteristics of Its Fuel Pressure. IERI Procedia, 2013,7:84-89

[5] A. Sala, B. Tormos, V. Macian. A Fuzzy Diagnosis Module for Oil Analysis in Industrial Diesel Engines. IFAC, 2004, 37(16):199-204

[6] B. Tormos, P. Olmeda. Analytical approach to wear rate determination for internal combustion engine condition monitoring based on oil analysis. Tribology International. 36(3):771-776

[7] B. Leal, J. Ordieres. Contaminants analysis in aircraft engine oil and its interpretation for the overhaul of the engine. Wseas Transactions on Information Science \& Applications, 2009, 6(10):1729-173

[8] C. Giancarlo, C. Ornella. Diagnostic methodology for internal combustion diesel engines via noise radiation. Energy Conversion and Management, 2015, 89:34-42

[9] J. Arroyo, F. Moreno. Diagnostic method based on the analysis of the vibration and acoustic emission energy for emergency diesel generators in nuclear plants. Applied Acoustics, 2013, 74(4):502-508

[10] Y. Cheng. Applications of Vibration Signal on Internal Combustion Engines Inspection. TIANJIN AUTO, 2008, 5: 48 49

[11] M. Desbazeille, R.B. Randall. Model-based diagnosis of large diesel engines based on angular speed variations of the crankshaft. Mechanical Systems and Signal Processing, 2010, 24:1529-1541

[12] D. Taraza, N.A. Henein, W. Bryzik, The frequency analysis of the crankshaft's speed variation: a reliable tool for diesel engine diagnosis, Journal of Engineering for Gas Turbines and Power, 2001,123(2):428432

[13] J. Zheng. Application of EMD in Internal Combustion Engine Troubleshooting. Internal Combustion Engines, 2012, 1:55 58 